\title{
A case of collapse
}

\author{
Aravinthan Varatharaj, Fraser Gordon and Peter Williams
}

\begin{abstract}
We describe the case of a woman who presented with seemingly non-specific symptoms, including collapse, but through examination was eventually revealed to have an unusual, potentially fatal, but treatable neurological condition. We share some thoughts on the process of diagnosis, the difficulty of diagnosing a rare disease and the practice of neurology on the acute medical take. We also highlight the value of screening neurological examination in acute medical patients.
\end{abstract}

KEY WORDS: collapse, neurology, heuristics

\section{Case presentation}

A 66-year-old woman presented to hospital following a collapse. She was previously fit and well, her only medical problems being stable hypertension and hypothyroidism. A week earlier, she had seen her own doctor after feeling 'flu-ish'. A few days later, she began to feel unsteady on her feet, 'like being drunk', and began to complain of 'seeing double'. Also, her daughter noticed that her speech was rather slurred. Subjectively, the patient felt that her left side was a little weak and that she 'drifted' to that side. The collapse occurred as she was walking around her home and she clearly remembered her legs 'giving way'. On examination, it was noted that she had dysarthria and that, although muscle power was normal in all limbs, she had bilateral upper limb dysmetria and was unable to walk in a straight line.

\section{What is the differential diagnosis?}

The symptoms were a subacute deterioration of global cerebellar function, with gait ataxia, bilateral upper limb dysmetria and dysarthria. In an older lady with hypertension, stroke would be a common cause of cerebellar dysfunction. For stroke, however, one would expect an acute history that has features referable to one vascular territory; for example, stroke in one cerebellar hemisphere typically results in ipsilateral dysmetria. Bilateral dysmetria caused by bilateral cerebellar hemisphere strokes is unusual but not implausible, especially with a cardiac source of emboli.

Neoplasia is another common cause of cerebellar dysfunction; in the older patient, this typically results from metastases from

Aravinthan Varatharaj, ${ }^{1}$ core medical trainee; Fraser Gordon, ${ }^{2}$ specialist registrar in geriatrics; Peter Williams, ${ }^{2}$ consultant in acute and emergency medicine

${ }^{1}$ North Middlesex University Hospital, London, UK; ${ }^{2}$ Whiston Hospital, Prescot, Merseyside, UK breast or lung cancers. Antibody-mediated paraneoplastic cerebellar degeneration can also occur in various cancers including lung, breast and ovarian.

In the younger patient, demyelinating diseases such as multiple sclerosis (MS) or acute disseminated encephalomyelitis (ADEM) would be another important differential, as would alcohol intoxication, which impairs cerebellar function and after prolonged use causes cerebellar degeneration. In addition, Wernicke's encephalopathy may occur as a result of thiamine deficiency in malnourished alcoholics; this classically presents as a triad of ataxia, ophthalmoplegia and confusion. Our patient did not consume alcohol and was not confused. She had not been exposed to drugs such as phenytoin or lithium, which may cause ataxia.

\section{Case progression}

A computed tomogram (CT) scan of the head was normal. Routine blood tests, electrocardiogram, and chest radiograph were also normal. A magnetic resonance (MR) scan with diffusion-weighted imaging (DWI) was performed and, again, was normal.

Meanwhile, the physiotherapists noted that the patient complained of worsening double vision. As the diagnosis remained unclear, the case was re-reviewed and a detailed neurological examination performed. This demonstrated complete paralysis of eye movements, profound upper and lower limb ataxia, global absence of reflexes and glove-and-stocking sensory loss.

A diagnosis of the Miller Fisher variant of Guillain-Barré syndrome was made, based on the triad of ophthalmoplegia, ataxia, and areflexia. Urgent spirometry was organised and revealed a forced vital capacity (FVC) of $0.81(11 \mathrm{ml} / \mathrm{kg}$; less than $20 \mathrm{ml} / \mathrm{kg}$ is considered an indication for ventilatory support). Given the impending respiratory failure, a decision was made for immediate intubation and ventilation. The patient was transferred to the Intensive Care Unit and commenced on a standard course of intravenous immunoglobulin (IVIg) for 5 days. Lumbar puncture was performed; the results of cerebrospinal fluid (CSF) analysis are as shown in Table 1. From here on, the patient made an excellent recovery, leaving hospital ten days later with normal limb power, co-ordination and speech.

\section{Discussion}

Miller Fisher syndrome is rare, with an estimated annual incidence of one per million, ${ }^{1}$ and is certainly not a typical cause of collapse in an older woman presenting to the acute medical unit. The key lesson from this case is the process of diagnosis. 
Table 1. Results of cerebrospinal fluid analysis.

\begin{tabular}{lll} 
Test & Result & Normal values \\
Appearance & Clear, colourless fluid & Clear, colourless fluid \\
CSF protein & $0.69 \mathrm{~g} / \mathrm{l}$ & $<0.5 \mathrm{~g} / \mathrm{l}$ \\
CSF glucose & $\begin{array}{l}4.7 \mathrm{mmol} / \mathrm{l}(63 \% \text { of } \\
\text { plasma glucose) }\end{array}$ & $>50 \%$ plasma glucose \\
CSF WCC & $<1 \mathrm{~mm}^{-3}$ & $<5$ lymphocytes $\mathrm{mm}^{-3}$ allowable \\
CSF RBC & $22 \mathrm{~mm}^{-3}$ & 0 , although cells present from \\
& & traumatic tap \\
\hline CSF = cerebrospinal fluid; $\mathrm{RBC}=$ red blood cell count; WCC = white cell count.
\end{tabular}

Our diagnosis of Miller Fisher syndrome was largely clinical, although several investigations provided additional evidence. The CSF finding of a slightly raised protein with normal cell count was suggestive, although CSF can often be normal in the first weeks of disease. ${ }^{2}$ Serum anti-GQ1B antibodies were positive, as is typical, ${ }^{3}$ although this test took weeks to return and was therefore not helpful in the acute setting. Viral serology was positive for cytomegalovirus IgM, suggesting recent infection and the cause of the preceding flu-like illness. Cytomegalovirus is a common immunological trigger for Guillain-Barré syndrome, ${ }^{4}$ although the result did not in itself confirm our diagnosis. Nerve conduction studies showed no significant motor conduction abnormalities but did show reduced sensory nerve action potentials, effectively excluding classical Guillain-Barré syndrome and supporting our diagnosis of the Miller Fisher variant. The single most important test that led to the correct diagnosis was not, however, any of these advanced investigations; it was the simple clinical finding of ophthalmoplegia.

Neurological examination is easily omitted or curtailed for acute medical patients who are admitted with a non-specific presentation. Realistically, it is often impractical to perform a full examination, especially when the patient is confused or uncooperative. Nevertheless, there are a few key bedside tests that can be performed relatively quickly and easily, even for patients who are unable to cooperate, and these should be routinely employed as a screening examination for neurological disease. Speech, eyes, face, limb power, co-ordination and reflexes are worth assessing in almost every case.

In acute medicine, diagnoses are usually formulated in one of two ways. ${ }^{5}$ Sometimes, a systematic assessment of findings generates a list of differential diagnoses, each of which can be
Key learning points.

- Common things are common, but many people have rare diseases

- Although a full neurological examination can sometimes be impractical, a few quick screening tests are of value in almost all acute medical patients

- A subacute presentation of neurological features that are not referable to one part of the brain is not typical of stroke

- Ataxia may masquerade as weakness, and vice versa

- Intuitive diagnosis is useful, but systematic diagnosis is helpful when a patient presents with a vague or atypical presentation, or when the underlying diagnosis is a rare disease

proved or disproved. In most cases, however, an intuitive diagnosis is rapidly made using triggers gained from the history and examination, which can then be confirmed using early investigation. Intuitive diagnosis using thinking short-cuts or 'heuristics' allows large numbers of acute, undifferentiated patients to be rapidly assessed and treated. Unfortunately, intuitive diagnosis can be prone to errors, particularly when a patient presents with atypical or vague symptoms that do not immediately suggest a diagnosis, or when the underlying disease is a rare one. On these occasions, we should shift from intuitive diagnosis formation to the slower but more systematic assessment of patients.

\section{References}

1 Schabet M. Miller Fisher syndrome. Pract Neurol 2009;9:289-91.

2 Nishimoto Y, Odaka M, Hirata K, Yuki N. Usefulness of anti-GQ1b IgG antibody testing in Fisher syndrome compared with cerebrospinal fluid examination. J Neuroimmunol 2004;148:200-5.

3 Yuki N, Sato S, Tsuji et al. Frequent presence of anti-GQ1b antibody in Fisher's syndrome. Neurology 1993;43:414-7.

4 Jacobs BC, Rothbarth PH, van der Meché FG et al. The spectrum of antecedent infections in Guillain-Barré syndrome: a case-control study. Neurology 1998;51:1110-5.

5 Kassirer JP, Wong JB, Kopelman RI. Learning clinical reasoning, 2nd edn. Philadelphia: Lippincott Williams \& Wilkins, 2009.

Address for correspondence: Dr A Varatharaj, Department of Geriatrics, North Middlesex University Hospital, Sterling Way, London N18 1QX.

E-mail: a.varatharaj@gmail.com 